Article

\title{
Prediction Model for the Anisotropic Thermal Conductivity of a 2.5-D Braided Ceramic Matrix Composite with Thin-Wall Structure
}

\author{
Zecan Tu ${ }^{1}$, Junkui Mao ${ }^{1,2, *}$, Xingsi Han ${ }^{1}$ and Zhenzong He ${ }^{1}$ \\ 1 Jiangsu Province Key Laboratory of Aerospace Power System, College of Energy and Power Engineering, \\ Nanjing University of Aeronautics and Astronautics, Nanjing 210016, China; tzc@nuaa.edu.cn (Z.T.); \\ xshan@nuaa.edu.cn (X.H.); hezhenzong@nuaa.edu.cn (Z.H.) \\ 2 Collaborative Innovation Center for Advanced Aero-Engine, Beijing 100191, China \\ * Correspondence: mjkpe@nuaa.edu.cn
}

Received: 31 January 2018; Accepted: 19 February 2019; Published: 1 March 2019

\begin{abstract}
The thickness of the hot component in a turbine engine is usually small. Therefore, the traditional prediction model of anisotropic thermal conductivity (ATC) based on the periodic hypothesis may be improper for use in the thermal analysis of ceramic matrix composite (CMC) components with a thin-wall structure. Thus, the prediction model for the ATC of a 2.5-D braided $\mathrm{CMC}$ was investigated, taking into account the actual thickness of the CMC thin-wall structure. An RVE (Representative Volume Element) model with a periodic boundary and a full-size model with the actual thickness were built to study the temperature field, the heat flux field, and the effective thermal conductivity of the CMC. A validation experiment was carried out to verify the accuracy of the two prediction models. The effect of the composite's thickness on the ATC and the critical thickness suitable for the RVE model were also studied. The results showed that in the thermal analysis of the thin-wall structure, the RVE model had a large deviation in the estimation of the effective thermal conductivity in the thickness direction. The relative error between the numerical data based on the RVE model and the experimental data reached $10.93 \%$, while the relative error was only $3.53 \%$ for the full-size model. Additionally, with increasing thickness, the effective thermal conductivities, based on the RVE model and the full-size model, were close to each other. For the critical thickness for the RVE model, which would be suitable for the prediction of the ATC, if the material's thermal properties such as the absolute value ratio and the level of anisotropy changed, the corresponding critical thickness was also different. For the ATC of the $\mathrm{SiC} / \mathrm{SiC}$ composites used in this study, the critical thickness was found to be $18.4 \mathrm{~mm}$, nearly 31 times larger than the RVE model's thickness.
\end{abstract}

Keywords: ceramic matrix composite; anisotropic thermal conductivity; 2.5-D braided composite; representative volume element; thin-wall structure

\section{Introduction}

The inlet temperatures of gas turbine engines are becoming higher in order to obtain better performances. Those high inlet temperatures could be far beyond the temperature limits of current superalloy materials [1]. More coolant and more complex cooling configurations need to be applied, but the performance of the turbine engines could be weakened. Consequently, highly heat-resistant materials, such as ceramic matrix composite (CMC), have attracted a great deal of attentions in recent years [2-4].

For the application of CMCs for the components with high temperatures, thermal analysis is an important research topic. Michael et al. [5] studied the temperature distribution of a CMC turbine vane 
with a film-cooling configuration, both experimentally and numerically, for a gas turbine environment. Their results encouraged the potential applications of CMC materials for turbine airfoil. Heidmann et al. [6] performed a numerical study of the heat transfer process of a nickel-based alloy vane case and a silicon nitride ceramic vane case in which the thermal conductivities were different. The results showed that the thermal conductivity had a significant effect on the internal heat transfer, which further affected the vane's temperature field.

However, as a fiber reinforced composite, the thermal conductivity of the CMC material is anisotropic $[7,8]$ due to the significant differences between the thermal conductivities in the fiber's axial direction and the radial direction and the differences between the thermal conductivities of the fibers and the matrix. Lebel et al. [9] investigated the thermal and mechanical performances of the $\mathrm{CMC}$ materials under the cyclic thermal stresses of a gas turbine combustion chamber. The anisotropic thermal conductivity (ATC) was used in the analysis, and the in-plane and the through-thickness thermal conductivities were set as $3 \mathrm{~W} /(\mathrm{m} \cdot \mathrm{K})$ and $1.8 \mathrm{~W} /(\mathrm{m} \cdot \mathrm{K})$, respectively. Tu and Mao et al. [10] studied the thermal analysis method of a CMC turbine vane. In the simulation, the effective ATCs were used to reflect the CMC's anisotropy. The results showed that the directions of the ATCs changed at different locations on the CMC turbine vane, and as a result, the braided direction was changed due to the warped surface. This variation of the ATC affected the heat transfer in the solid region of the CMC turbine vane. Liu et al. [11] carried out a multi-scale thermodynamic analysis for a CMC turbine vane. The in-plane and the through-thickness thermal conductivities were $12.1 \mathrm{~W} /(\mathrm{m} \cdot \mathrm{K})$ and $8.83 \mathrm{~W} /(\mathrm{m} \cdot \mathrm{K})$, respectively. The variation of the material's ATC local coordinates due to the vane's curved surface was also considered.

The research mentioned above indicates that in the thermal analysis of a CMC component with a high temperature, the ATC is an important parameter. However, the ATC varies for different CMC materials, and the internal structure and the percentages of the components with different thermal properties have significant influences on the ATC. For example, Kiani et al. [12] studied the thermal conductivity of triaxial braided composites with fibers that changed directions. The results showed that when the direction of the fibers changed from $\pm 25^{\circ}$ to $\pm 65^{\circ}$, the thermal conductivity in the thickness direction changed from $4.2 \mathrm{~W} /(\mathrm{m} \cdot \mathrm{K})$ to $5.15 \mathrm{~W} /(\mathrm{m} \cdot \mathrm{K})$. In the work of Jiang et al. [13], the thermal conductivities of 3-D braided composites were investigated. The results showed that the volume fraction of the fibers had a considerable effect on the thermal conductivity. When the volume fraction of the fibers increased from 0.25 to 0.55 , the thermal conductivity in the braided direction changed from $0.68 \mathrm{~W} /(\mathrm{m} \cdot \mathrm{K})$ to $1.3 \mathrm{~W} /(\mathrm{m} \cdot \mathrm{K})$.

Thus, the prediction method of the braided CMC's ATC is an important aspect in the application of the CMC, which has been widely studied. The homogenization method [14], the thermoelectric analogy model [15], and the mathematical model [16] have been developed for many years, and they have different advantages depending on the purpose. The Representative Volume Element (RVE) method was developed based on the homogenization method, and it has attracted much attention due to the fact that it could introduce many information of the micro/meso-structures. This model was firstly studied by Hill [17]. A simple way to apply the RVE model is to use the minimal structural repeating unit with a periodicity hypothesis. For example, Siddiqui et al. [18] investigated the effective thermal conductivity and thermal resistance of a 2-D woven fabric using the finite element method. An RVE model including two warp yarns and two weft yarns was applied based on the scanning electron microscope, and the geometrical size of the RVE was $0.862 \mathrm{~mm} \times 0.862 \mathrm{~mm} \times 0.5 \mathrm{~mm}$. Ai et al. [19] studied the thermal conductivity of 3-D woven C/C composites at high temperature numerically and experimentally. The multi-scale method was applied, including the microscale model and the mesoscale model, in which the mesoscale model was an RVE model including two warp yarns, two weft yarns, and one yarn in the thickness direction. The geometrical size of the RVE was $1.96 \mathrm{~mm}$ $\times 1.96 \mathrm{~mm} \times 0.76 \mathrm{~mm}$, and the thickness of the experimental sample was $5 \mathrm{~mm}$. Fang et al. [20] built an RVE model with a geometrical size of $2.724 \mathrm{~mm} \times 2.724 \mathrm{~mm} \times 5.843 \mathrm{~mm}$ to predict the thermal 
conductivities of three-dimensional four-directional braided composites. Similar works can also be found in other references [21-23].

All of the studies mentioned above used an RVE model where the geometrical structure was based on the periodicity hypothesis and the global thickness of the RVE models was in the range of $0.5 \mathrm{~mm}$ to $3 \mathrm{~mm}$. However, the thickness of the hot components, such as the turbine vane, was small $(2-3 \mathrm{~mm})$. Therefore, for the thermal analysis of a CMC component and the prediction of the thermal conductivity of the corresponding material, the RVE model may be improper. The periodicity hypothesis, which is the foundation of the RVE model, is unsatisfied.

Some researchers tried to build a model with a full size in the thickness direction. For example, Dong et al. [24] numerically and experimentally investigated the thermal conductivity of 2.5-D angle-interlock woven composites. A full-size model with a geometrical size of $8.8 \mathrm{~mm} \times 8.46 \mathrm{~mm} \times$ $6.26 \mathrm{~mm}$ was built. In this model, the thickness of the fiber bundles was nearly $0.3 \mathrm{~mm}$. The results showed that the thermal conductivity in the thickness direction based on the full-size model agreed well with the experimental data, and the relative error was about $4.1 \%$. Although the full-size model could describe the geometrical characteristics more accurately, it needed much more meshes than the RVE model, due to the big differences between the sizes of the microstructure and the macroscopic components. This limited the application of the full-size model in the thermal analysis of the CMC components with large sizes. Therefore, there is a critical thickness for the application of the RVE model or the full-size model, which need to be studied considering both the accuracy and the efficiency.

Accordingly, the present work aims to investigate the ATC prediction model of the 2.5-D braided ceramic matrix composites, taking into account the actual thickness of the high-temperature components. An RVE model with a periodic boundary and a full-size model based on the actual thickness were built to study the effect of the composite thickness on the estimation accuracy of the effective thermal conductivity. The validation experiment was carried out to verify the estimation method of the effective thermal conductivity. Additionally, the influence of the thickness on the effective thermal conductivity and the critical thickness that was suitable for the RVE model were also studied under different operating conditions of the CMC's ATC.

\section{Research Models}

Figure 1a shows a photograph of the 2.5-D braided $\mathrm{CMC}(\mathrm{SiC} / \mathrm{SiC})$. According to the information supplied by the material supplier SAFEI group, the axial and the radial thermal conductivities of the $\mathrm{SiC}$ fiber bundle are $9.66 \mathrm{~W} /(\mathrm{m} \cdot \mathrm{K})$ and $1.48 \mathrm{~W} /(\mathrm{m} \cdot \mathrm{K})$, and the thermal conductivity of the ceramic matrix is $6.5 \mathrm{~W} /(\mathrm{m} \cdot \mathrm{K})$. The sample used in the numerical simulation and the experiment are shown in Figure 1. The size of this sample was $10 \mathrm{~mm} \times 10 \mathrm{~mm} \times 2.8 \mathrm{~mm}$ (length $\times$ width $\times$ height).

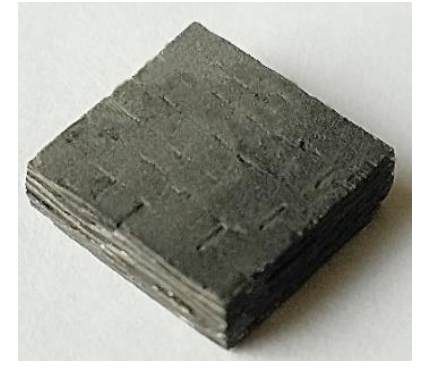

(a) Photograph of the 2.5-D braided CMC sample

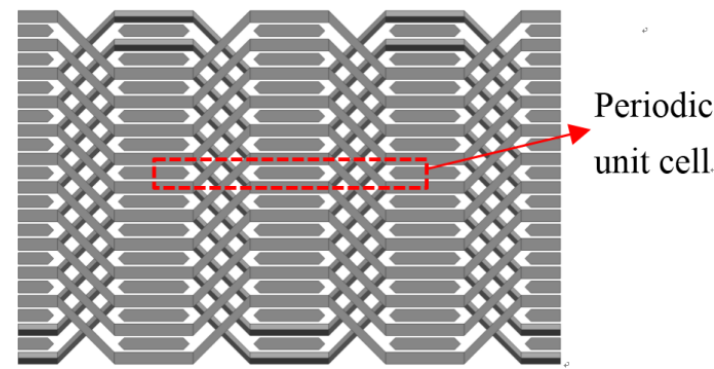

(b) A schematic diagram of the braided structure

Figure 1. (a) A photograph and (b) a schematic diagram of the 2.5-D braided ceramic matrix composite (CMC).

Figure $1 \mathrm{~b}$ shows a schematic diagram of the ideal structure of the 2.5-D braided fiber bundles. A minimal periodic unit cell was created as the RVE model, which was applied in the thermal analysis of the 2.5-D braided CMC. Similar to the RVE model in Reference [25], an RVE model was built of 
the 2.5-D braided CMC, considering the geometry characteristics of the 2.5-D braided CMC shown in Figure 1a. For the model, the following hypotheses are considered:

1. The sections of the axial yarns and the braided yarns are both hexagons.

2. There are no cracks in the models. Instead, they are completely continuous.

Figure 2 shows the RVE model of the 2.5-D braided CMC. The size of the model was $5.72 \mathrm{~mm} \times$ $3.34 \mathrm{~mm} \times 0.6 \mathrm{~mm}$ (length $\times$ width $\times$ height), as shown in Figure 2a. According to the data of the scanning electron microscope test, the average size of the sections of the fiber bundles was $1.5 \mathrm{~mm} \times$ $0.25 \mathrm{~mm}$ (width $\times$ height), as shown in Figure $2 \mathrm{~b}$. The angle of the braided yarns was $45^{\circ}$. The parts of the matrix and the fiber bundles were given in Figure 2c,d.

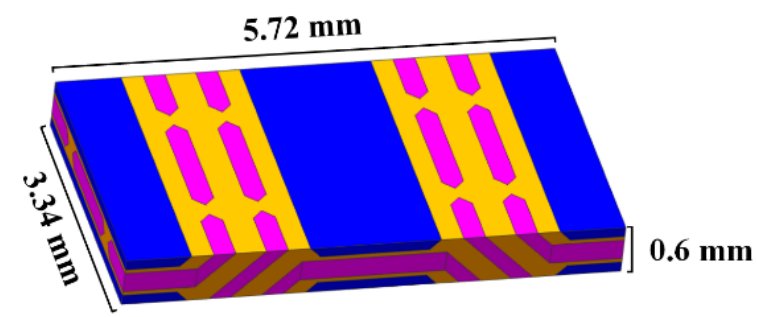

(a) The RVE model

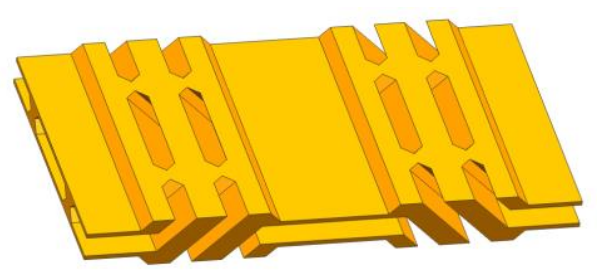

(c) The matrix

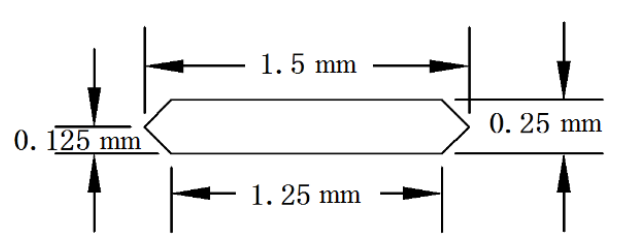

(b) The section of the fiber bundles

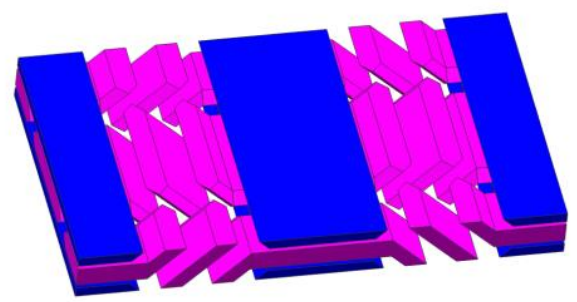

(d) The fiber bundles

Figure 2. A schematic diagram of the Representative Volume Element (RVE) model.

Additionally, a full-size model was built to compare with the RVE model. Figure 3 shows the full-size model, which was built based on the actual size of the sample shown in Figure 1a. The geometry size was $11.4 \mathrm{~mm} \times 10 \mathrm{~mm} \times 2.8 \mathrm{~mm}$ (length $\times$ width $\times$ height), for which the length was set as $11.4 \mathrm{~mm}$ to preserve the periodicity of side surfaces. The distances between the two adjacent axial yarns on the $z$-axis and $x$-axis were $0.6 \mathrm{~mm}$ and $1.4 \mathrm{~mm}$, respectively. The distance between the two adjacent braided yarns on the $y$-axis was $0.2 \mathrm{~mm}$. The angle of the braided yarns was $45^{\circ}$.

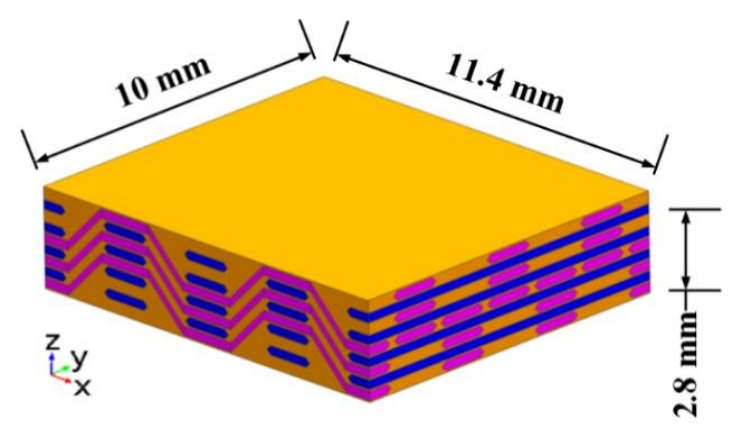

(a) The full-size model
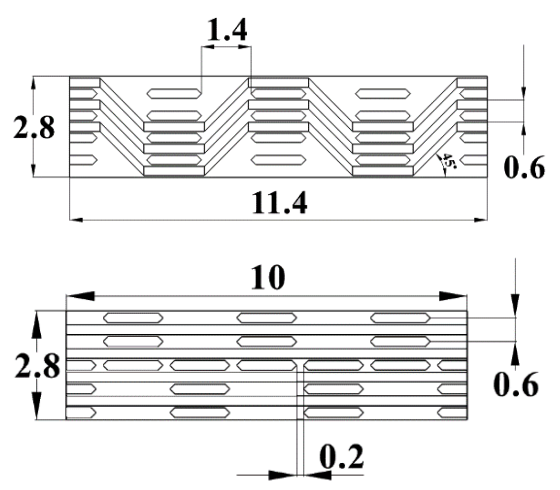

(b) The sections of the full-size model

Figure 3. Cont. 


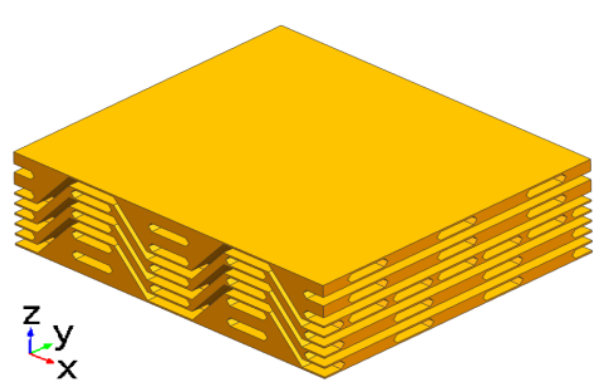

(c) The matrix

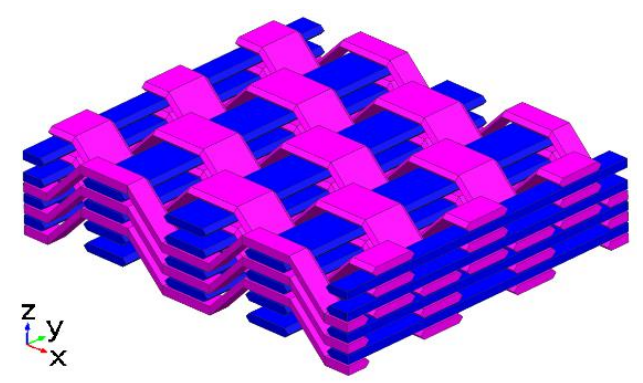

(d) The fiber bundles

Figure 3. Schematic diagrams of the full-size model.

\section{Numerical Methodology}

For the two numerical models mentioned above, i.e., the RVE model and the full-size model, the thermal analysis was carried out using the Finite Element Method (FEM) based on the Comsol software. In the simulation, the thermal conductivity of the matrix and the fiber bundles were firstly calculated, with particular attention to the ATCs of different parts of the braided yarn due to the varied directions of the fiber bundles. Then, the meshes of the two models were generated, and the boundary conditions with constant temperatures were applied. Finally, the finite element simulation of the temperature fields was carried out. The information about the heat flux and the thermal gradient was obtained simultaneously, and this information was used to calculate the effective thermal conductivity. The steps of the procedure are given below.

\subsection{Governing Equations}

In the solid region, the steady-state energy transport equation in the tensor form is written as [26]

$$
\rho C_{p} \vec{v} \cdot \nabla T=\nabla \cdot\left(k_{i j} \nabla T\right)+Q
$$

where $C_{p}$ is the specific heat capacity at constant pressure and $Q$ is the source term. The term on the left-hand side represents the convective energy transfer due to the rotational or translational motion of the solids. The velocity field, $\vec{v}$, is computed from the motion specified for the solid zone. In this paper, both this term and $Q$ are zero because the solid region is stationary. $k_{i j}$ is the thermal conductivity, which is anisotropic in this paper.

\subsection{Application of the ATC}

In the RVE model shown in Figure 2, the thermal conductivity of the matrix is isotropic, but the thermal conductivity of the fiber bundles is anisotropic, and the thermal conductivity in the axial direction is bigger than that in the radial direction. To represent the ATC of the fiber bundles, a local Cartesian coordinate system $(\zeta, \eta, v)$ was introduced, with the $\zeta$ axis along the yarn's axial direction. The $\zeta$ axis was considered as the principal direction of the thermal conductivity (PDTC) because the thermal conductivity was largest in this direction.

The axial yarn's direction was along the $Y$ axis of the global Cartesian coordinate system $(X, Y, Z)$, as shown in Figure 4, so the ATC of axial yarns could be represented by three thermal conductivities on the $X, Y$, and $Z$ axes, respectively. However, the directions of the braided yarns were changed in different parts, as shown in Figure 4. There was an inclined angle between the local Cartesian coordinate $(\zeta, \eta, v)$ and the global Cartesian coordinate $(X, Y, Z)$, so the ATC applied for $(X, Y, Z)$ needed to be transferred, and the transformation method was described as follows. 


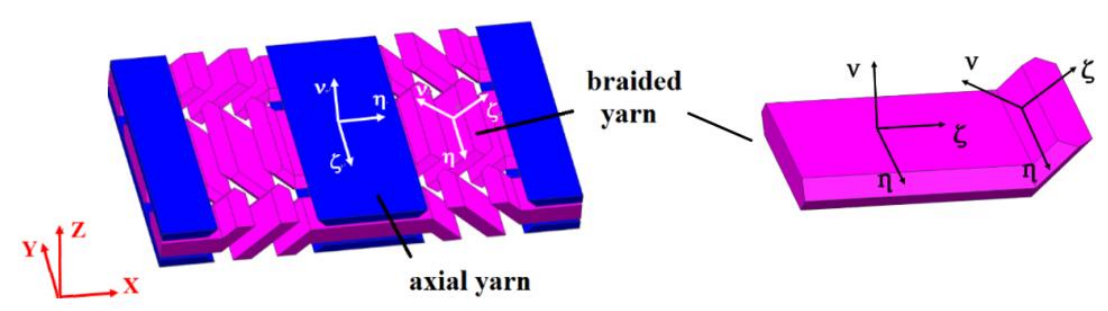

Figure 4. A schematic diagram of the principal direction of the thermal conductivity (PDTC).

According to the study in Reference [27], the anisotropic thermal conductivity $k_{i j}$ can be expressed as the matrix

$$
k_{i j}=\left[\begin{array}{lll}
k_{X X} & k_{X Y} & k_{X Z} \\
k_{Y X} & k_{Y Y} & k_{Y Z} \\
k_{Z X} & k_{Z Y} & k_{Z Z}
\end{array}\right]
$$

Equation (2) can be rewritten as shown below:

$$
\left[\begin{array}{lll}
k_{X X} & k_{X Y} & k_{X Z} \\
k_{Y X} & k_{Y Y} & k_{Y Z} \\
k_{Z X} & k_{Z Y} & k_{Z Z}
\end{array}\right]=\left[\begin{array}{lll}
l_{1} & m_{1} & n_{1} \\
l_{2} & m_{2} & n_{2} \\
l_{3} & m_{3} & n_{3}
\end{array}\right]\left[\begin{array}{ccc}
k_{\zeta} & 0 & 0 \\
0 & k_{\eta} & 0 \\
0 & 0 & k_{v}
\end{array}\right]\left[\begin{array}{ccc}
l_{1} & l_{2} & l_{3} \\
m_{1} & m_{2} & m_{3} \\
n_{1} & n_{2} & n_{3}
\end{array}\right]
$$

where

$$
\left[\begin{array}{lll}
l_{1} & m_{1} & n_{1} \\
l_{2} & m_{2} & n_{2} \\
l_{3} & m_{3} & n_{3}
\end{array}\right]=\left[\begin{array}{ccc}
\cos \gamma \cos \beta & -\cos \alpha \sin \gamma+\cos \gamma \sin \beta \sin \alpha & \sin \gamma \sin \alpha+\cos \gamma \sin \beta \cos \alpha \\
\sin \gamma \cos \alpha & \cos \gamma \cos \alpha+\sin \gamma \sin \beta \sin \alpha & -\cos \gamma \sin \alpha+\sin \gamma \sin \beta \cos \alpha \\
-\sin \beta & \cos \beta \sin \alpha & \cos \beta \cos \alpha
\end{array}\right]
$$

Equation (4) shows the transform matrix between the local coordinate system (principal direction of the thermal conductivity) and the global Cartesian coordinate system, with the inclined PDTC angles $\alpha, \beta$ and $\gamma$.

\subsection{Mesh and Boundary Conditions}

The top and bottom surfaces of the RVE model and the full-size model on the $z$-axes were applied with constant temperature boundary conditions. The temperatures on the top and bottom surfaces were set as $273 \mathrm{~K}$ and $283 \mathrm{~K}$, respectively. The four side surfaces were applied with periodic boundary conditions, as shown in Figure $5 \mathrm{a}, \mathrm{b}$.

To generate the mesh, a grid dependency test based on the RVE model was carried out. The maximum mesh size was changed from $1.09 \mathrm{~mm}$ to $0.2 \mathrm{~mm}$, and the corresponding numbers of the meshes were 9490, 17639, 41295, 71,898, 110,014, and 209,159. Figure $5 \mathrm{c}$ shows the variation of $\mathrm{k}_{\mathrm{Z}}$ with the increasing number of the mesh. The results show that when the number was equal to 71,898 , the relative variation of $\mathrm{k}_{\mathrm{Z}}$ was smaller than $1 \%$.

Finally, the number of the mesh applied for the RVE model was 71,898 . The maximum and minimal mesh sizes were $0.458 \mathrm{~mm}$ and $0.057 \mathrm{~mm}$, and the mesh growth rate was smaller than 1.45 , as shown in Figure 5a. For the full-size model, the mesh generation strategy was the same as that of the RVE model. The corresponding number of the mesh was 798,492, the maximum and minimal mesh sizes were kept at $0.458 \mathrm{~mm}$ and $0.057 \mathrm{~mm}$, and the mesh growth rate was smaller than 1.45 , as shown in Figure 5b.

\subsection{Operating Conditions and Parameters Definition}

In the simulation, there were two kinds of numerical models, i.e., the RVE model and the full-size model. The RVE model was a minimal periodic element in the 2.5-D braided CMC, while the full-size model was based on the sample's real size for the 2.5-D braided CMC. 


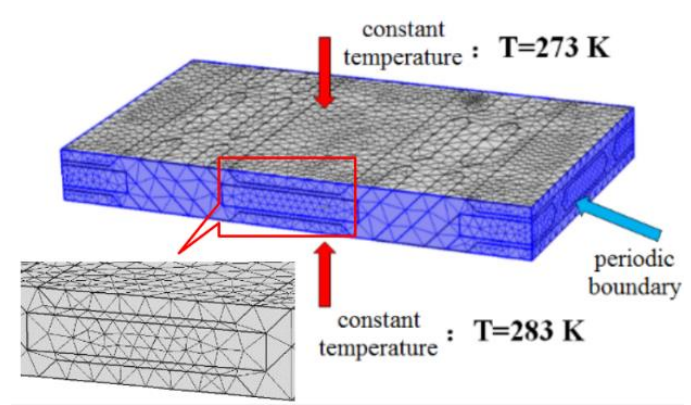

(a) RVE model

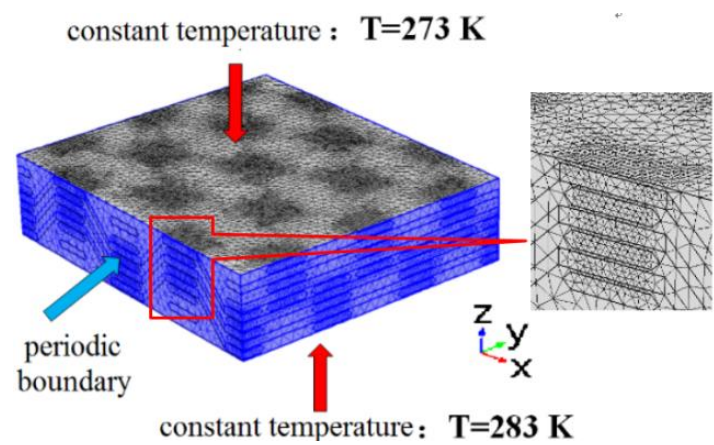

(b) Full-size model

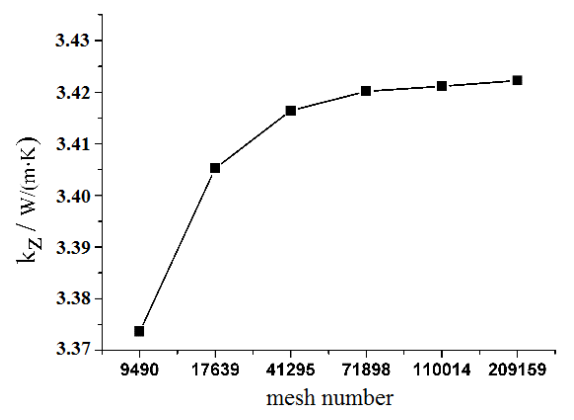

(c) Grid dependency test

Figure 5. The mesh and boundary conditions.

Simultaneously, to study the influence of the sample thickness on the Effective Thermal Conductivity (ETC) and the critical thickness that was suitable for the RVE model, full-size models with different thickness of the $z$-axis were built. The thicknesses $L_{Z}$ were set as $2.8 \mathrm{~mm}, 4.0 \mathrm{~mm}$, $7.6 \mathrm{~mm}, 11.2 \mathrm{~mm}, 14.8 \mathrm{~mm}$, and $18.4 \mathrm{~mm}$, as shown in Figure 6. The boundary conditions and the mesh generation strategies were the same for the model shown in Figure $5 \mathrm{~b}$. The corresponding numbers of the meshes were $798,492,1,297,051,2,785,965,4,264,916,5,465,846$, and 6,760,725, respectively.

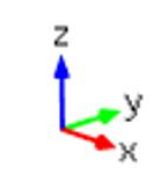

$2.8 \mathrm{~mm}$

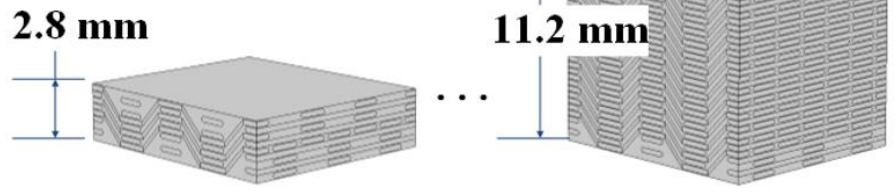

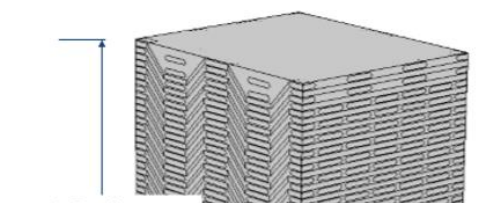

$18.4 \mathrm{~mm}$

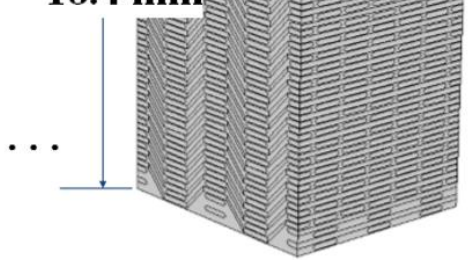

Figure 6. Full-size models with different thicknesses.

Additionally, to make the results and conclusions more useful, in the investigation of the thermal conductivities with increasing thickness, the absolute value ratio, $\mathrm{n}$ (as shown in Equation (5)), and the level of anisotropy, $r$ (as shown in Equation (6)), of the thermal conductivities of the fiber bundles were changed.

$$
\begin{gathered}
\mathrm{n}=\frac{k_{\zeta}}{9.66}=\frac{k_{\eta}}{1.48}=\frac{k_{v}}{1.48} \\
r=\frac{k_{\zeta}}{k_{\eta}}=\frac{k_{\zeta}}{k_{v}}
\end{gathered}
$$


where $k_{\zeta}, k_{\eta}$, and $k_{v}$ are the thermal conductivities along the $\zeta, \eta$, and $v$ directions and their values are $9.66 \mathrm{~W} /(\mathrm{m} \cdot \mathrm{K}), 1.48 \mathrm{~W} /(\mathrm{m} \cdot \mathrm{K})$, and $1.48 \mathrm{~W} /(\mathrm{m} \cdot \mathrm{K})$, respectively, of the sample in Figure 1.

The operating conditions of the absolute value ratio $\mathrm{n}$ and the level of anisotropy $r$ were set according to the data in Reference [28], as shown in Table 1. The ATC of the sample shown in Figure 1a was set as the basic Case 1 . For Cases 2-5, the values of $k_{\zeta}, k_{\eta}$, and $k_{v}$ were increased at the same time to improve the absolute value ratio $\mathrm{n}$ and the level of anisotropy $\mathrm{r}$ was kept constant. For Cases $6-9$, the $k_{\eta}$ and $k_{v}$ were kept constant and the $k_{\zeta}$ was increased to improve the level of anisotropy $r$. Correspondingly, the absolute value $\mathrm{n}$ did not exist. The corresponding $k_{\zeta}, k_{\eta}$, and $k_{v}$ for each case are also given in Table 1.

Table 1. A summary of the cases studied.

\begin{tabular}{cccc}
\hline Case & $k_{\zeta} / k_{\eta} / k_{v} \mathrm{~W} /(\mathbf{m} \cdot \mathrm{K})$ & $n$ & $\boldsymbol{r}$ \\
\hline 1 & $9.66 / 1.48 / 1.48$ & 1 & 6.53 \\
2 & $19.32 / 2.96 / 2.96$ & 2 & 6.53 \\
3 & $38.64 / 5.92 / 5.92$ & 4 & 6.53 \\
4 & $57.96 / 8.88 / 8.88$ & 6 & 6.53 \\
5 & $96.6 / 14.8 / 14.8$ & 10 & 6.53 \\
6 & $14.8 / 1.48 / 1.48$ & $/$ & 10 \\
7 & $19.24 / 1.48 / 1.48$ & $/$ & 13 \\
8 & $23.68 / 1.48 / 1.48$ & $/$ & 16 \\
9 & $29.6 / 1.48 / 1.48$ & $/$ & 20 \\
\hline
\end{tabular}

In the analysis of the results, the parameter $F_{f}$ that represents the volume fraction of the fibers is defined as follows:

$$
F_{f}=\frac{V_{f}}{V_{t}}
$$

where $V_{f}$ is the volume of fibers and $V_{t}$ is the total volume of the model.

Additionally, although the constant temperature boundary conditions in different models were the same, it should be noted that the thickness changed, so the temperature and the heat flux in different models varied. To compare the temperature and the heat flux in different models fairly, the relative temperature fluctuations $\delta_{T}$ and the relative heat flux fluctuations $\delta_{h f}$ are defined:

$$
\begin{gathered}
\delta_{T}=\frac{T_{i}-T_{a v g}}{10[\mathrm{~K}]} \\
\delta_{h f}=\frac{h f_{i}-h f_{a v g}}{h f_{a v g}}
\end{gathered}
$$

where $T_{i}$ is the local temperature, $T_{\text {avg }}$ is the average temperature, and $10 \mathrm{~K}$ is the difference between the two constant temperature boundary conditions. The $h f_{i}$ term is the local heat flux, and $h f_{\text {avg }}$ is the average heat flux.

When the relative deviation between the ETCs based on the RVE model and the full-size model was smaller than $5 \%$, the corresponding thickness of the full-size model was defined as the "critical thickness", $\mathrm{L}_{\mathrm{Z}}$. For different ATC cases in Table 1, the critical thickness $\mathrm{L}_{\mathrm{Z}}$ which was suitable for the RVE model was investigated in this paper.

\section{Results and Discussions}

\subsection{Comparison between the RVE Model and the Full-Size Model}

To analyze the difference between the results based on the RVE model and the full-size model, the temperature fields, the heat flux fields, and the effective thermal conductivity of the two models were analyzed. In the simulation, the ATC was set as the basic Case 1, and the thickness of the 
full-size model was the sample's actual size $(2.8 \mathrm{~mm})$. Additionally, tests of the sample's thermal conductivity through the thickness were carried out to validate the accuracy of the RVE model and the full-size model.

\subsubsection{Temperature Field}

Figure 7 shows the temperature fields of the RVE model and the full-size model. The temperature distribution of the RVE's surface is shown in Figure 7a. In the figure, regions A and C are the matrix and region $B$ is the fiber bundles. It can be seen that the temperature distributions of the three regions had significant differences. In the regions between the fiber bundles and the matrix (A and B; B and C), the directions of the isotherms had considerable variations. The reason for this was that in the process of heat transfer from the high-temperature surface to the low-temperature surface (via the red arrows shown in Figure 7a), there were different regions with different thermal conductivities, which led to heat conduction along multiple walls. In the fiber bundles, the horizontal yarns and the diagonal yarns had different directions, which led to different ATCs, so the transmission paths of the heat in the fiber bundles also varied.

Figure $7 \mathrm{~b}$ shows the temperature distribution on the surface of the full-size model. The temperature field was also heterogeneous. There were significant thermal gradients in the region between the fiber bundles and the matrix, which was similar to the RVE model. The reason for this was also that the transmission path of the heat changed due to the difference of the fibers' and the matrix's thermal conductivities.

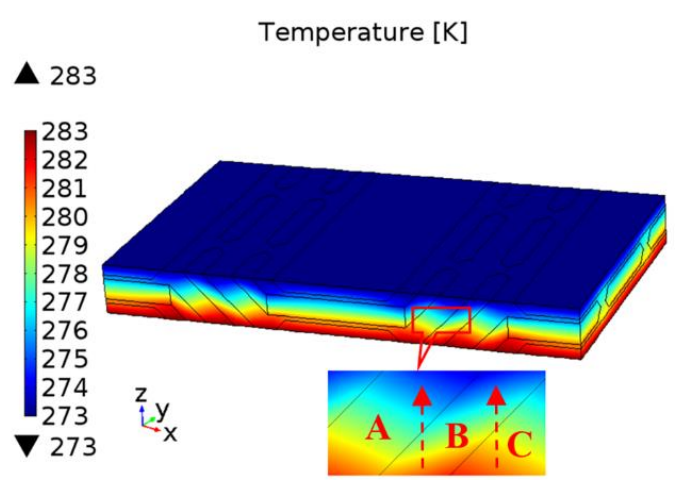

(a) Surface of the RVE model

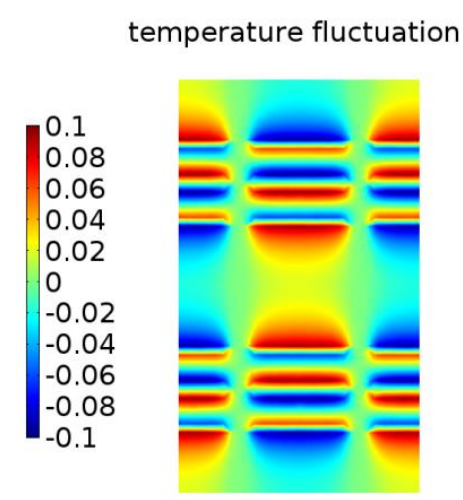

(c) Middle section of the RVE model

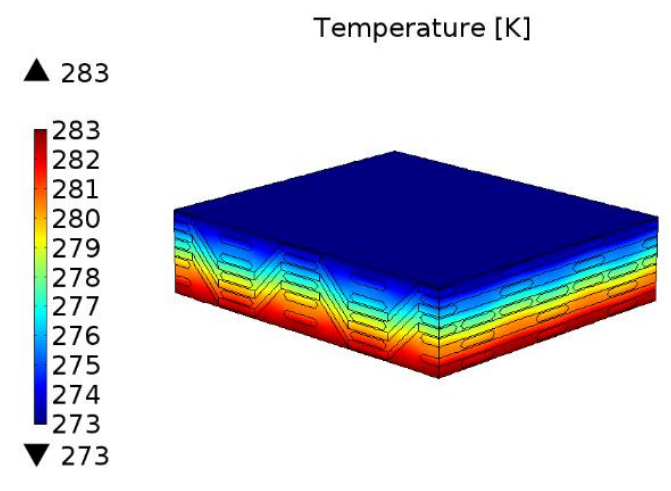

(b) Surface of the full-size model

temperature fluctuation
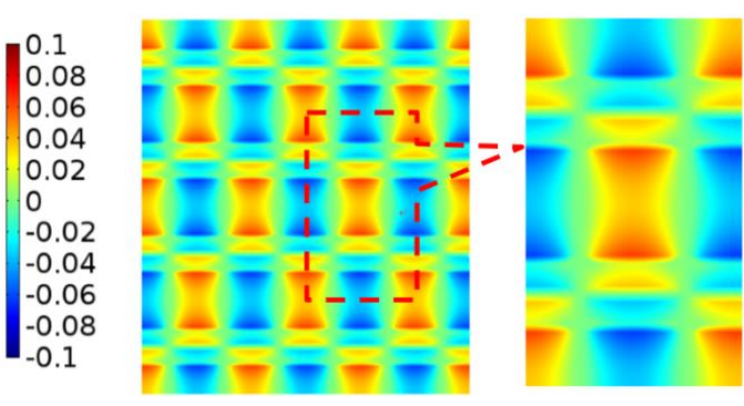

(d) Middle section of the full-size model

Figure 7. The temperature fields.

Figure $7 \mathrm{c}$,d shows the distributions of the temperature fluctuation $\delta_{T}$ in the middle sections of the $z$-axes of the two models. The results show that $\delta_{T}$ for these sections was obvious in both the fiber bundles and the matrix. Additionally, in the middle section of the full-size model, $\delta_{T}$ of the part with 
the same size of the RVE model was found. The distributions of $\delta_{T}$ in those two models had significant differences, and $\delta_{T}$ in the RVE model had more dramatic changes compared to $\delta_{T}$ in the full-size model. For the RVE model, the maximum $\delta_{T}$ in the middle section was $9.96 \%$, while for the full-size model, the maximum $\delta_{T}$ in the middle section was $6.39 \%$.

\subsubsection{Heat Flux Field}

To analyze the influence of ATC on the heat transfer in the 2.5-D braided CMC, Figure 8 shows the heat flux distributions of the RVE model and the full-size model. From Figure 8a,b, it can be seen that the heat flux fields were heterogeneous and that the heat flux of the diagonal braided yarns was larger than the heat flux of the axial yarns on the $Y$-axis and the horizontal braided yarns on the $x$-axis. The reason for this was that the axial and radial thermal conductivities of the fibers bundle were $9.66 \mathrm{~W} /(\mathrm{m} \cdot \mathrm{K})$ and $1.48 \mathrm{~W} /(\mathrm{m} \cdot \mathrm{K})$, and the thermal conductivity of the matrix was $6.5 \mathrm{~W} /(\mathrm{m} \cdot \mathrm{K})$. In the diagonal braided yarns, there was a component of the bigger thermal conductivity $(9.66 \mathrm{~W} /(\mathrm{m} \cdot \mathrm{K}))$ on the $z$-axis, leading to an enhancement of the heat conduction on the $z$-axis.

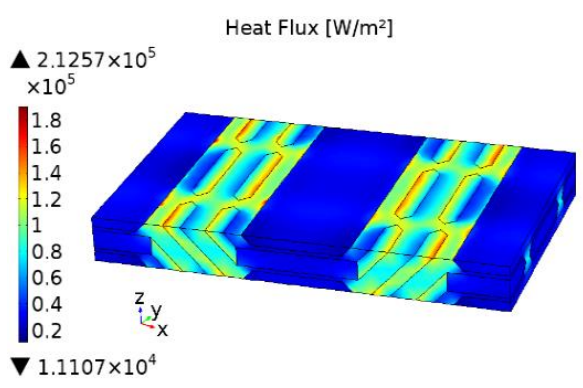

(a) Surface of the RVE model heatflux fluctuation

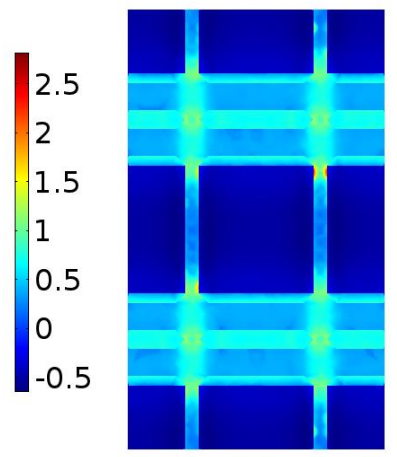

(c) Middle section of the RVE model

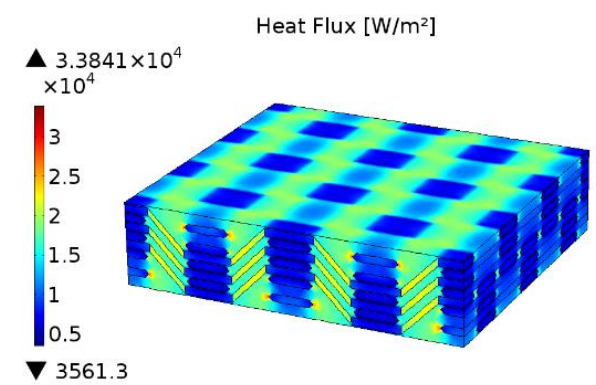

(b) Surface of the full-size model heatflux fluctuation

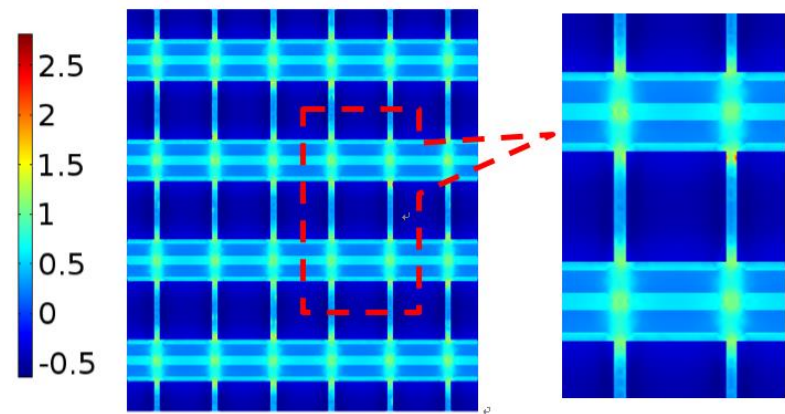

(d) Middle section of the full-size model

Figure 8. The heat flux fields of the RVE model.

Figure $8 \mathrm{c}$,d shows the distributions of the heat flux fluctuation $\delta_{h f}$ for the middle sections of the $\mathrm{Z}$ axes of the two models. The results show that the heat flux fluctuations were significant and that the distributions for those two models were similar. For the RVE model, the maximum $\delta_{h f}$ was $255.58 \%$. For the full-size model, the maximum $\delta_{h f}$ reached $280.40 \%$.

\subsubsection{Effective Thermal Conductivity}

According to the results mentioned above, the average heat flux on the $z$-axis of the RVE model was $56,958 \mathrm{~W} / \mathrm{m}^{2}$, the difference between the constant temperatures of top and bottom surfaces was $10 \mathrm{~K}$, and the thickness of the RVE model was $0.6 \mathrm{~mm}$. In the full-size model, the average heat flux on the $z$-axis was $13,968 \mathrm{~W} / \mathrm{m}^{2}$, the difference between the constant temperatures of the top and bottom 
surfaces was $10 \mathrm{~K}$, and the thickness was $2.8 \mathrm{~mm}$. Therefore, the effective thermal conductivities on the $\mathrm{z}$-axes $\mathrm{k}_{\mathrm{Z}}$ of the two models could be calculated based on Fourier's thermal conduction equation.

The $\mathrm{k}_{\mathrm{Z}}$ values of the RVE model and the full-size model were $3.42 \mathrm{~W} /(\mathrm{m} \cdot \mathrm{K})$ and $3.91 \mathrm{~W} /(\mathrm{m} \cdot \mathrm{K})$, respectively. The ETC $\mathrm{k}_{Z}$ based on the RVE model and the ETC $\mathrm{k}_{Z}$ based on full-size model had a significant difference. The reason for this was that the thickness of the full-size model was small $(2.8 \mathrm{~mm})$ and the periodicity was unsatisfied in the $\mathrm{Z}$ axis direction for the full-size model, leading to a large difference for the periodic RVE model.

Furthermore, the effective thermal conductivities along the $x$ - and $y$-axes ( $\mathrm{k}_{\mathrm{X}}$ and $\mathrm{k}_{\mathrm{Y}}$ ) could also be calculated using a similar method. The $\mathrm{k}_{X}$ values of the RVE model and full-size model were $5.63 \mathrm{~W} /(\mathrm{m} \cdot \mathrm{K})$ and $5.62 \mathrm{~W} /(\mathrm{m} \cdot \mathrm{K})$, respectively, and the $\mathrm{k}_{\mathrm{Y}}$ values of the two models were $5.28 \mathrm{~W} /(\mathrm{m} \cdot \mathrm{K})$ and $5.77 \mathrm{~W} /(\mathrm{m} \cdot \mathrm{K})$, respectively. A comparison between the ATCs based on the RVE model and full-size model is shown in Figure 9.

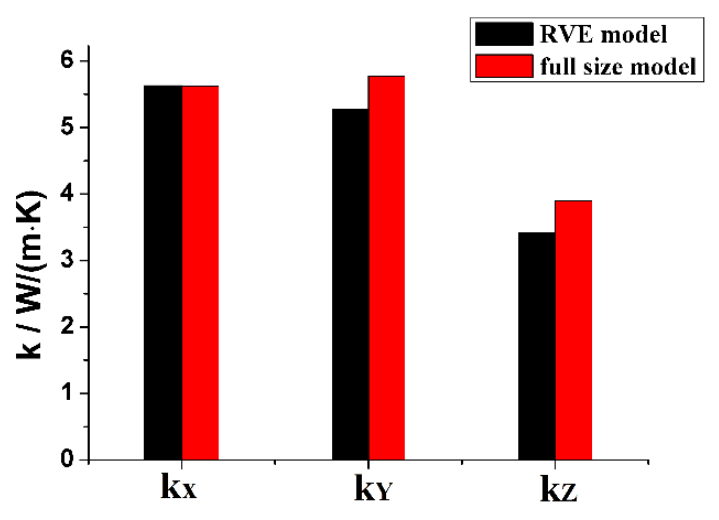

Figure 9. The effective thermal conductivities based on the two models.

\subsubsection{Validation of the RVE Model and the Full-Size Model}

To validate the accuracies of the RVE model and the full-size model, the thermal conductivity of the $\mathrm{z}$-axis $\mathrm{k}_{\mathrm{Z}}$ for the sample shown in Figure 1a was tested. A NETZSCH LFA 457 Micro-Flash instrument was used to measure the thermal diffusion coefficient, and the specific heat capacity was measured with a DSC $200 \mathrm{~F} 3$. The geometrical sizes of the samples were $10 \mathrm{~mm} \times 10 \mathrm{~mm} \times 2.8 \mathrm{~mm}$ (length $\times$ width $\times$ height), which satisfied the demands of the test equipment. Finally, the thermal conductivity was calculated using the density, the thermal diffusivity, and the specific heat capacity. In the experiment, the $\mathrm{k}_{\mathrm{Z}}$ values at four different positions of the sample were tested.

According to the experimental results, the density of the sample was $1.711 \times 10^{3} \mathrm{~kg} / \mathrm{m} 3$ and the specific heat capacity was $0.688 \times 10^{3} \mathrm{~J} /(\mathrm{kg} \cdot \mathrm{K})$. Table 2 shows the experimental data of the thermal diffusivity and the corresponding thermal conductivity on the $\mathrm{Z}$ axis. The thermal conductivities at four different positions on the sample were tested. The results show that the four different thermal conductivities were close to each other. The average $\mathrm{k}_{\mathrm{Z}}$ value was $4.053 \mathrm{~W} /(\mathrm{m} \cdot \mathrm{K})$.

Table 2. A comparison of the experimental and numerical data.

\begin{tabular}{|c|c|c|c|c|c|c|c|c|c|c|c|}
\hline \multicolumn{9}{|c|}{ Experimental Data } & \multicolumn{3}{|c|}{ Numerical Data } \\
\hline \multicolumn{4}{|c|}{ Thermal Diffusivity $/ \mathrm{m}^{2} / \mathrm{s}$} & \multicolumn{4}{|c|}{$\begin{array}{c}\text { Thermal Conductivity } \\
k_{\mathrm{Z}} / \mathrm{W} /(\mathrm{m} \cdot \mathrm{K})\end{array}$} & $\begin{array}{c}\text { Mean } \\
\text { Value/W/(m·K) }\end{array}$ & $\begin{array}{c}\text { Standard } \\
\text { Deviation } / \mathrm{W} /(\mathrm{m} \cdot \mathrm{K})\end{array}$ & \multirow{2}{*}{$\begin{array}{c}\begin{array}{c}\text { RVE } \\
\text { Model/W/(m·K) }\end{array} \\
3.42\end{array}$} & \multirow{2}{*}{$\begin{array}{c}\begin{array}{c}\text { Full-Size } \\
\text { Model/W/(m·K) }\end{array} \\
3.91\end{array}$} \\
\hline 3.427 & 3.460 & 3.444 & 3.443 & 4.036 & 4.072 & 4.053 & 4.052 & 4.053 & 0.015 & & \\
\hline
\end{tabular}

The numerical results in Section 4.1.3 are also given in Table 2. The numerical results of the $\mathrm{k}_{\mathrm{Z}}$ values based on the RVE model and the full-size model are $3.42 \mathrm{~W} /(\mathrm{m} \cdot \mathrm{K})$ and $3.91 \mathrm{~W} /(\mathrm{m} \cdot \mathrm{K})$, respectively, and the relative differences compared to the experimental data are $15.62 \%$ and $3.53 \%$, respectively. The numerical result based on the full-size model is closer to the experimental data. In the thermal analysis of thin-walled structures such as a turbine vane, the RVE model would lead to a large 
deviation in the estimation of the effective thermal conductivity. The reason for this is that the main difference between the RVE model and the full-size model is the boundary part shown in Figure 10. The periodic hypothesis of the RVE model could not be satisfied in the CMC's boundary parts, and the volume fractions of the fibers and the braided structure are also different, which would lead to the variation of the heat transfer in these parts. In the RVE model and the full-size model, the volume fractions of the fibers $F_{f}$ were $54.34 \%$ and $39.61 \%$, respectively. Therefore, the full-size model could truly reflect the internal structure of a CMC thin-wall structure.

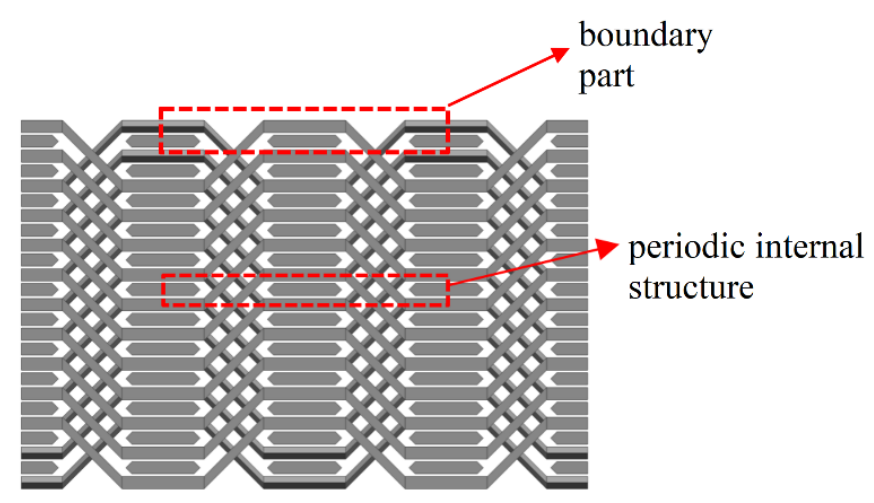

Figure 10. A comparison between the boundary part and the periodic internal structure.

\subsection{Influence of the Thickness}

Although the full-size model can obtain more accurate results in comparison to the RVE model, it needs too many computing resources when the geometric size is large. The influence of the thickness on the ETC and the critical thickness that was suitable for the RVE model are analyzed in this section.

Figure 11a shows the variation curve of the $\mathrm{k}_{Z}$ with increasing the thickness $\mathrm{L}_{Z}$. It can be seen that $\mathrm{k}_{Z}$ firstly decreased and then tended to be constant. When $\mathrm{L}_{Z}$ reached $18.4 \mathrm{~mm}$, the relative variation of the $k_{Z}$ was only $0.51 \%$, which could be treated as convergent. When the $L_{Z}$ was equal to $2.8 \mathrm{~mm}$ and $18.4 \mathrm{~mm}$, the corresponding $\mathrm{k}_{\mathrm{Z}}$ values were $3.91 \mathrm{~W} /(\mathrm{m} \cdot \mathrm{K})$ and $3.53 \mathrm{~W} /(\mathrm{m} \cdot \mathrm{K})$, respectively, and the relative variation was $10.76 \%$. The results show that the thickness had a big influence on the ETC $\mathrm{k}_{\mathrm{Z}}$ of the full-size model. The reason for this was that the volume fraction of the fibers $\mathrm{V}_{\mathrm{f}}$ increased with increasing $\mathrm{L}_{Z}$, as shown in Figure 11b. For example, when $\mathrm{L}_{Z}$ was equal to $2.8 \mathrm{~mm}$ and $18.4 \mathrm{~mm}$, the corresponding $\mathrm{V}_{\mathrm{f}}$ values were $39.61 \%$ and $51.92 \%$, respectively. The variation of $\mathrm{V}_{\mathrm{f}}$ changed the percentage of the components with different thermal properties, which further affected the ETC of the full-size model.

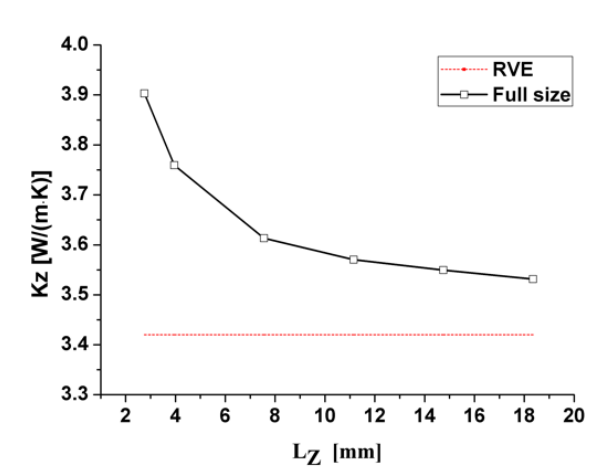

(a) $\mathrm{kz}$

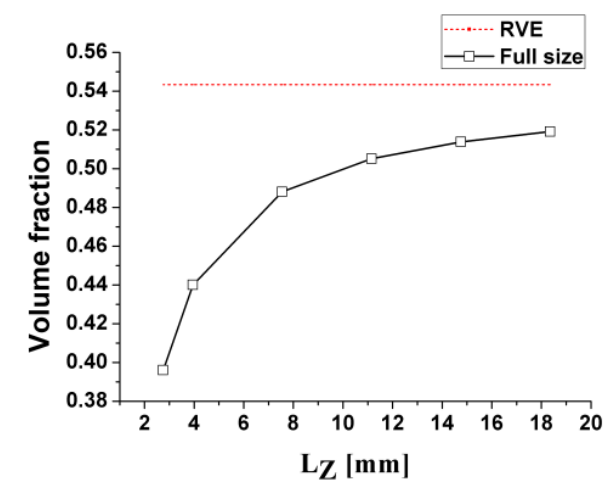

(b) Volume fraction $F_{f}$

Figure 11. The curves of the $\mathrm{k}_{\mathrm{Z}}$ and the volume fraction with the $\mathrm{L}_{\mathrm{Z}}$ increasing.

At the same time, according to the results in Section 4.1.3, the ETC $\mathrm{k}_{\mathrm{Z}}$ based on the RVE model was $3.42 \mathrm{~W} /(\mathrm{m} \cdot \mathrm{K})$, as shown by the red dashed line in Figure 11. It can be seen that the line based on the 
full-size model tended to converge on the red dashed line of the RVE model. When $\mathrm{L}_{Z}$ changed from 2.8 $\mathrm{mm}$ to $18.4 \mathrm{~mm}$, the relative variation between the results based on the full-size model and RVE model varied from $12.53 \%$ to $3.12 \%$. The results indicate that when the thickness $\mathrm{L}_{Z}$ was large, the ETC $\mathrm{k}_{Z}$ based on the RVE model and the ETC $\mathrm{k}_{Z}$ based on the full-size model were close to each other. The reason for this was that when the $\mathrm{L}_{Z}$ was large enough, the periodicity was approximately satisfied in the z-axis direction for the full-size model, leading to a small difference with the periodic RVE model and that the $F_{f}$ of these two models were close to each other. For example, $F_{f}$ of the RVE model and $F_{f}$ of the full-size model with $\mathrm{L}_{Z}=18.4 \mathrm{~mm}$ were $54.34 \%$ and $51.92 \%$, respectively, with a relative variation of only $4.45 \%$. For a full-size model with $\mathrm{L}_{Z}=2.8 \mathrm{~mm}$, this relative variation reached $27.11 \%$.

For the operating condition discussed in this section, when $\mathrm{L}_{Z}$ was bigger than $18.4 \mathrm{~mm}$, the relative variation was smaller than $5 \%$, so the ETC $k_{Z}$ based on the RVE model could represent the result based on the full-size model. The corresponding Lz of $18.4 \mathrm{~mm}$ was determined as the "critical thickness", which was nearly 31 times greater than the thickness of the RVE model.

\subsection{Results with Different ATCS}

The results discussed above were based on the 2.5-D braided CMC sample, as shown in Figure 1. In the engineering application, the material's ATCs were different if the internal components and the structure were changed. To make the present results and conclusions more applicable, the influence of the thickness and the critical thickness that were suitable for the RVE model were further investigated under different operating conditions of the ATCs.

Figure 12 shows the thermal conductivities $\mathrm{k}_{\mathrm{Z}}$ based on the RVE model and the full-size model with an increasing thickness $\mathrm{L}_{Z}$, when the absolute value ratio $\mathrm{n}$ and the level of anisotropy $r$ of the thermal conductivities of the fiber bundles were changed.

The results in Figure 12a show that when the values of $k_{\zeta}, k_{\eta}$, and $k_{v}$ were different, the influence of the thickness was also changed. In Case 1 and Case 2, the value of $k_{Z}$ decreased with the increase of $L_{Z}$, while in Cases $3-5, k_{Z}$ increased with the increase of $L_{Z}$. Figure $12 b$ gives the influence law of the level of anisotropy $\mathrm{r}$. It can be seen that when $\mathrm{r}$ increased, the $\mathrm{k}_{\mathrm{z}}$ 's variation curves always monotonically decreased, but the range became smaller. The reason for this was that the main difference between the RVE model and the full-size models was the existence of the boundary parts and that its influence was affected by the relative variations between the thermal conductivities of the fiber bundles and the matrix, which changed with the absolute value ratio $n$ and the level of anisotropy $r$.

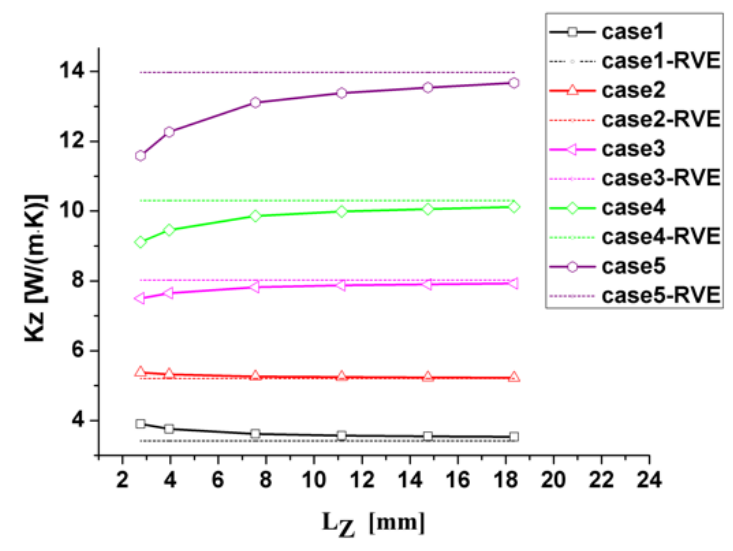

(a) The absolute value ratio $\mathrm{n}$

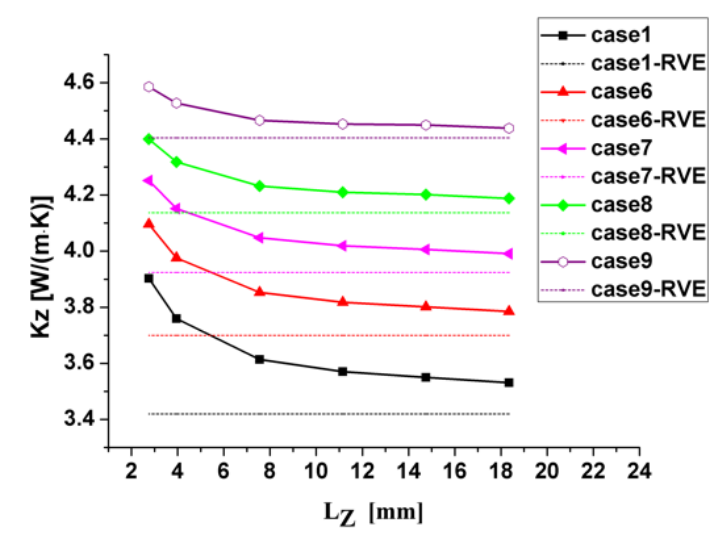

(b) The level of anisotropy $r$

Figure 12. The variations of the thermal conductivities $\mathrm{k}_{\mathrm{Z}}$ with different ATCs.

Additionally, the results in Figure 12 show that when the absolute value ratio and the level of anisotropy changed, the lines based on the full-size models always tended to converge on the corresponding dash lines of the RVE models. This phenomenon was explained in Section 4.2. In this paper, the convergence criteria was set to that standard, and the relative variation between the results 
based on the RVE model and the full-size model was smaller than 5\%. Figure 13 shows the variation of the "critical thickness" $\mathrm{L}_{Z}$ under different ATCs. The results show that when the absolute value ratio $\mathrm{n}$ increased, the "critical thickness" $\mathrm{L}_{\mathrm{Z}}$ first decreased and then increased. When the absolute value ratios were 1,2, 4, 6, and 10, the corresponding critical thicknesses were $11.2 \mathrm{~mm}, 2.8 \mathrm{~mm}, 4.0 \mathrm{~mm}$, $7.6 \mathrm{~mm}$, and $11.2 \mathrm{~mm}$, respectively. When the level of anisotropy $\mathrm{r}$ increased, the "critical thickness" Lz decreased almost monotonously. When the levels of anisotropy $r$ were 6.53, 10, 13, 16, and 20, the corresponding critical thicknesses were $11.2 \mathrm{~mm}, 7.6 \mathrm{~mm}, 7.6 \mathrm{~mm}, 4.0 \mathrm{~mm}$, and $2.8 \mathrm{~mm}$, respectively.

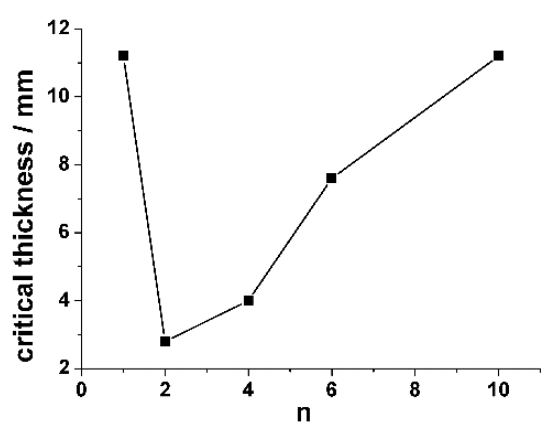

(a) The absolute value ratio $\mathrm{n}$

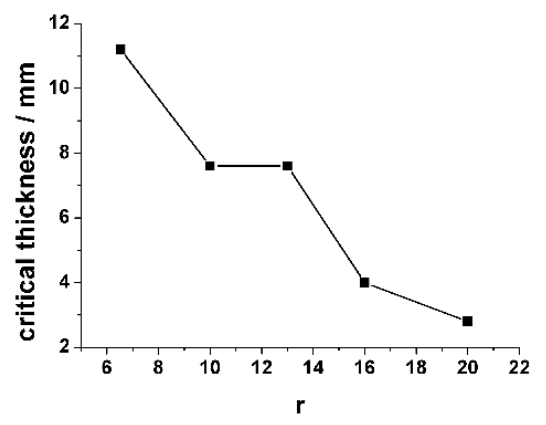

(b) The level of anisotropy $r$

Figure 13. The variations of the critical thickness with different ATCs.

In general, the structure of the boundary part of the braided CMC, which was different for a periodic internal structure, was the main reason for the difference between the results of the full-size model and the RVE model. This influence depended on the value and the level of anisotropy of the ATC, i.e., the relative difference between the thermal conductivities of the matrix and the fibers.

\section{Conclusions}

A numerical study of the anisotropic thermal conductivities, especially the thermal conductivity in the thickness direction, of the 2.5-D braided CMC material was carried out. Two prediction models, an RVE model with a periodicity hypothesis and a full-size model with actual thickness, were established. An experimental test of the thermal conductivity in the thickness direction was also used to validate these two prediction models. The influence of the thickness on the ATC and the critical thickness that was suitable for the RVE model were further investigated under different operating conditions of the thermal properties of the fibers. The main observations can be summarized as follows:

(1) The temperature field and the heat flux field inside the 2.5-D braided CMC material were clearly heterogeneous, and these fields were affected by the difference between the thermal conductivities of the fiber bundles and matrix. For example, in the full-size model, the relative fluctuation of the temperature field and the relative fluctuation of the heat flux field in the middle section reached $6.39 \%$ and $280.40 \%$, respectively.

(2) In the thermal analysis of a thin-walled structure, such as a turbine vane, the RVE model would lead to a large deviation in the estimation of the effective thermal conductivity so that the periodic hypothesis could not be satisfied. The relative variation of the thermal conductivity based on the RVE model compared with the experimental data was $15.62 \%$, while the relative variation was only $3.53 \%$ when the full-size model was applied.

(3) When the thickness increased, the effective thermal conductivities based on the RVE model and the full-size model were close to each other. For the ATC of the sample used in this study, when the thickness was bigger than the critical thickness of $18.4 \mathrm{~mm}$, the RVE model was suitable for the prediction of the ATC.

(4) When the absolute value ratio and the level of anisotropy of the thermal conductivities of the fiber bundle were changed, the influence of the thickness on the thermal conductivity was different and the critical thickness for the RVE model changed. When the absolute value ratio increased, 
the critical thickness firstly decreased and then increased, and the critical thickness decreased almost monotonously with increasing the level of anisotropy.

Author Contributions: Conceptualization, Z.T. and J.M.; methodology, Z.T.; software, Z.T., X.X.; validation, Z.H. and X.H.; formal analysis, Z.T.; investigation, Z.T.; resources, Z.T.; data curation, Z.T.; writing-original draft preparation, Z.T.; writing — review and editing, X.H.; visualization, Z.H.; supervision, J.M.; project administration, J.M.; funding acquisition, Z.T., Z.H. and X.H.

Funding: This research was funded by the China Postdoctoral Science Foundation, grant number 2018M642248, the National Natural Science Foundation of China, grant number 51806103 and the Jiangsu Provincial Natural Science Foundation of China, grant number BK20160794.

Acknowledgments: Xingsi Han acknowledges the support of the Jiangsu Specially-Appointed Professor Program.

Conflicts of Interest: The authors declare no conflict of interest.

\section{Nomenclature}

$C_{p} \quad$ Specific heat capacity at constant pressure $(\mathrm{J} /(\mathrm{kg} \cdot \mathrm{K}))$

$\mathrm{F} \quad$ Volume fraction

$k \quad$ Thermal conductivity $(\mathrm{W} /(\mathrm{m} \cdot \mathrm{K}))$

L Thickness (mm)

$\mathrm{n} \quad$ Absolute value ratio comparing to the sample's ATC

$\mathrm{r} \quad$ Anisotropy's level

$\mathrm{T} \quad$ Temperature $(\mathrm{K})$

$\vec{v} \quad$ Velocity $(\mathrm{m} / \mathrm{s})$

$X, Y, Z \quad$ Global Cartesian coordinates

Greek symbols

$\alpha \quad$ Rotation angles around the $x$-axis between the PDTC coordinates and the global coordinates $\left({ }^{\circ}\right)$

$\beta \quad$ Rotation angles around the $y$-axis between the PDTC coordinates and the global coordinates $\left({ }^{\circ}\right)$

$\gamma \quad$ Rotation angles around the $z$-axis between the PDTC coordinates and the global coordinates $\left(^{\circ}\right)$

$\rho \quad$ Density $\left(\mathrm{kg} / \mathrm{m}^{3}\right)$

$\zeta, \eta, v \quad$ Local Cartesian coordinates

$\delta \quad$ Relative fluctuation

Subscripts

$f \quad$ Fiber

ij Coordinates of the mesh nodes

\section{Abbreviations}

$\begin{array}{ll}\text { ATC } & \text { Anisotropic Thermal Conductivity } \\ \text { CMC } & \text { Ceramic Matrix Composite } \\ \text { ETC } & \text { Effective Thermal Conductivity } \\ \text { FEM } & \text { Finite Element Method } \\ \text { PDTC } & \text { Principal Direction of Thermal Conductivity } \\ \text { RVE } & \text { Representative Volume Element }\end{array}$

\section{References}

1. Fang, C.D. Development Research of Aeroengine; Aviation Industry Press: Beijing, China, 2009.

2. Hiroshi, K. The application of ceramic-matrix composites to the automotive ceramic gas turbine. Compos. Sci. Technol. 1999, 59, 861-872.

3. Deng, Y.; Li, W.G.; Wang, R.Z. The temperature-dependent fracture models for fiber-reinforced ceramic matrix composites. Compos. Struct. 2016, 140, 534-539. [CrossRef]

4. Yang, J.S.; Dong, S.M.; Xu, C.Y. Mechanical response and microstructure of 2D carbon fiber reinforced ceramic matrix composites with $\mathrm{SiC}$ and Ti3SiC2 fillers. Ceram. Int. 2016, 42, 3019-3027. [CrossRef] 
5. Michael, V.; Anthony, C.; Robinson, R.C.; Thomas, D.J. Ceramic Matrix Composite Vane Subelement Testing in a Gas Turbine Environment; ASME: New York, NY, USA, 2004.

6. Heidmann, J.D.; Kassab, A.J.; Divo, E.A.; Rodriguez, F.; Steinthorsson, E. Conjugate Heat Transfer Effects on a Realistic Film-Cooled Turbine Vane; ASME: New York, NY, USA, 2003.

7. Behzad, T.; Sain, M. Measurement and prediction of thermal conductivity for hemp fiber reinforced composites. Polym. Eng. Sci. 2007, 10, 977-983. [CrossRef]

8. Xu, Y.B.; Yagi, K. Automatic FEM model generation for evaluating thermal conductivity of composite with random materials arrangement. Comput. Mater. Sci. 2004, 30, 242-250. [CrossRef]

9. Lebel, L.; Turenne, S.; Boukhili, R. An experimental apparatus and procedure for the simulation of thermal stresses in gas turbine combustion chamber panels made of ceramic matrix composites. J. Eng. Gas Turbines Power 2017, 139, 091502. [CrossRef]

10. Tu, Z.C.; Mao, J.K.; Jiang, H.; Han, X.; He, Z. Numerical method for the thermal analysis of a ceramic matrix composite turbine vane considering the spatial variation of the anisotropic thermal conductivity. Appl. Therm. Eng. 2017, 127, 436-452. [CrossRef]

11. Xin, L.I.U.; Xiuli, S.H.E.N.; Longdong, G.O.N.G.; Peng, L.I. Multi-scale thermodynamic analysis method for 2D SiC/SiC composite turbine guide vanes. Chin. J. Aeronaut. 2018, 31, 117-125.

12. Kiani, S.; Chan, W.S.; Sheikh, A.H. Measurement of thermal conductivity of triaxial braided composites. J. Compos. Mater. 2008, 42, 12. [CrossRef]

13. Jiang, L.L.; Xu, G.D.; Cheng, S. Predicting the thermal conductivity and temperature distribution in 3D braided composites. Compos. Struct. 2014, 108, 578-583. [CrossRef]

14. Dasgupta, A.; Agarwal, R.K. Orthotropic thermal conductivity of plain-weave fabric composites using a homogenization technique. J. Compos. Mater. 1992, 26, 2736-2758. [CrossRef]

15. Ning, Q.G.; Chou, T.W. Closed-form solutions of the in-plane effective thermal conductivities of woven-fabric composites. Compos. Sci. Technol. 1995, 55, 41-48. [CrossRef]

16. Bhattacharjee, D.; Kothari, V.K. A theoretical model to predict the thermal resistance of plain woven fabrics. Indian J. Fibre Text. Res. 2005, 30, 252-257.

17. Hill, R. Elastic properties of reinforced solids: Some theoretical principles. J. Mech. Phys. Solids 1963, 11, 357-372. [CrossRef]

18. Siddiqui, M.O.R.; Sun, D.M. Finite element analysis of thermal conductivity and thermal resistance behavior of woven fabric. Comput. Mater. Sci. 2013, 75, 45-51. [CrossRef]

19. Ai, S.G.; He, R.J.; Pei, Y.M. A numerical study on the thermal conductivity of 3D woven C/C composites at high temperature. Appl. Compos. Mater. 2015, 22, 823-835.

20. Fang, W.Z.; Chen, L.; Gou, J.J.; Tao, W.Q. Predictions of effective thermal conductivities for three-dimensional four-directional braided composite using the lattice Boltzmann method. Int. J. Heat Mass Transfer 2016, 92, 120-130. [CrossRef]

21. Islam, M.R.; Pramila, A. Thermal conductivity of fiber reinforced composites by FEM. J. Compos. Mater. 1999, 33, 1699-1715. [CrossRef]

22. Klett, J.W.; Ervin, V.J.; Edie, D.D. Finite-element modeling of heat transfer in carbon/carbon composites. Compos. Sci. Technol. 1999, 59, 593-607. [CrossRef]

23. Car, E.; Zalamea, F.; Oller, S.; Miquel, J.; Qnate, E. Numerical simulation of composite materials: Two procedures. Int. J. Solid Struct. 2002, 39, 1967-1986. [CrossRef]

24. Dong, K.; Liu, K.; Pan, L.J.; Gu, B.H.; Sun, B.Z. Experimental and numerical investigation on the thermal conduction properties of 2.5D angle-interlock woven composites. Compos. Struct. 2016, 154, 319-333. [CrossRef]

25. Guan, T.R. Micro Geometry and Mechanical Model and Experimental Study of 2.5D Braided Quartz/SiO2 Ceramic Matrix Composites; Nanjing University of Aeronautics and Astronautics: Nanjing, China, 2012.

26. COMSOL. COMSOL Multiphysics Help-Theory for the Heat Transfer, Release 4.3, Sweden. 2011.

27. Ree, Y.; Lin, J.R.; Zhu, J.G. Coordinate transformation uncertainty analysis in Larger-Scale metrology. IEEE Trans. Instrum. Meas. 2015, 64, 2380-2388.

28. He, F. Carbon Fiber and Graphite Fiber; Chemical Industry Press: Beijing, China, 2010.

(C) 2019 by the authors. Licensee MDPI, Basel, Switzerland. This article is an open access article distributed under the terms and conditions of the Creative Commons Attribution (CC BY) license (http:/ / creativecommons.org/licenses/by/4.0/). 